\title{
Analysis of Fault Diagnosis for Current and Vibration Signals in Pumps and Motors using a Reconstructed Phase Portrait
}

\section{]jfis}

Young-Ok Jung ${ }^{1}$ and Youngchul Bae ${ }^{2}$

${ }^{1}$ Department of Electrical and Semiconductor Engineering, Chonnam National University, Yeosu, Korea

${ }^{2}$ Division of Electrical, Electronic Communication and Computer Engineering, Chonnam National

University, Yeosu, Korea

\begin{abstract}
In this paper, we measure the current and vibration signals of one-dimensional time series that occur in a motor and pump, respectively. These machines are representative rotary and pumping machines. We also eliminate unnecessary components such as noise by preprocessing the current and vibration signals. Then, in order to diagnose fault signals for the pump and motor, we transform from one-dimensional time series to a two-dimensional phase portrait using Takens' embedding method. After this transformation, we review the variation in the pattern according to the fault signals.
\end{abstract}

Keywords: Fault diagnosis, Embedding method, chaos. Phase portrait

\section{Introduction}

The modern industrial complex typically includes various motors and pumps. Maintenance of these motors and pumps is of utmost importance in the industrial field. Typically, a motor or pump fault brings many failures in production and causes secondary troubles, including material damage and harm to workers. Therefore, it is very important to detect fault signals in motors and pumps before failure occurs.

During the past few decades, various studies of fault diagnosis have been conducted for motors and pumps [1-11].

Generally, the fault signs of motor and pump are judged by current and vibration signals.

Received: Aug. 17, 2015

Revised : Sep. 24, 2015

Accepted: Sep. 24, 2015

Correspondence to: Youngchul Bae

(ycbae@chonnam.ac.kr)

@The Korean Institute of Intelligent Systems

(c) This is an Open Access article distributed under the terms of the Creative Commons Attribution Non-Commercial License (http://creativecommons.org/licenses/ by-nc/3.0/) which permits unrestricted noncommercial use, distribution, and reproduction in any medium, provided the original work is properly cited.
In this paper, we measure the current and vibration signals of one-dimensional time series that occur in a motor and pump, respectively. These are representatives of rotary and pumping machines and are used for diagnosing faults. We also eliminate unnecessary component such as noise by preprocessing the current and vibration signals. Then, in order to diagnose fault signs for the pump and motor, we transform from the one-dimensional time series to a twodimensional phase portrait using Takens' embedding method [12]. After this transformation, we review the variation of pattern according to fault sign.

\section{Data Acquisition of Current and Vibration Signals in Motors and Pumps}

\subsection{Configuration of the Data Acquisition System}




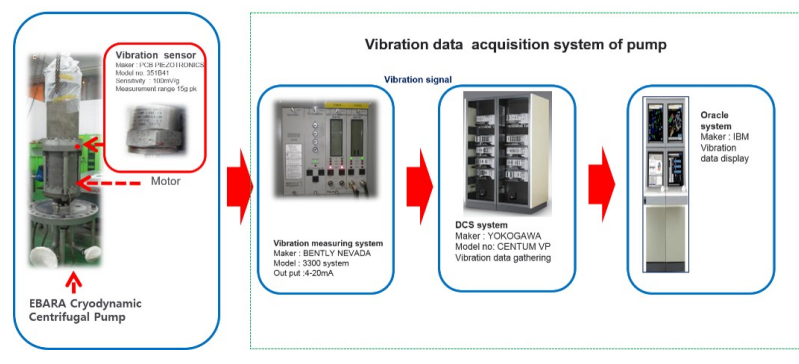

Figure 1. Data acquisition system for vibration.

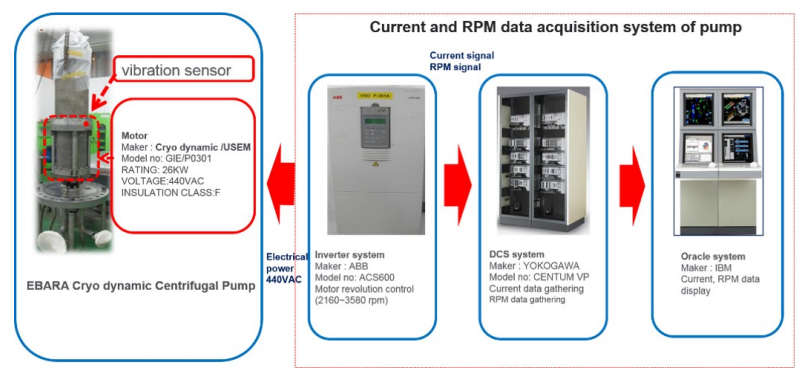

Figure 2. Data acquisition system for current and RPM.

Figures 1 and 2 show the data acquisition system to acquire the current, RPM, and vibration signals from a motor and pump system. We use a seven-stage EBARA Cryodynamic centrifugal pump. This pump operates at temperatures as low as $-150^{\circ} \mathrm{C}$, with a rated rotational speed of $3580 \mathrm{rpm}$ and a power supply of 440 VAC using an inverter.

This data acquisition system consists primarily of four systems, including the current and RPM measuring system for the motor, vibration measuring system for the pump, distributed control system (DCS), and analysis tool system.

The vibration measuring system (model 330, GE Bently Nevada, Minden, NV, USA) has an output current of 4-20 mA.

The motor is made by Cryodynamic/USEM. This 26-kW motor operates at 440 VAC and has class F insulation.

The current and RPM measuring system consists of an inverter, a DCS, and an analyzing tool. The inverter system was made by ABB and has a resolution control of 2160-3580 RPM.

The CENTUM VP DCS system, made by Yokogawa, Japan, gathers vibration data from pumps and motors.

The IBM analysis tool system displays the current and vibration data in the main console. We do not use the function of the analysis tool in this study because we need only onedimensional data of the motor and pump in order to apply chaotic theory and analyze the fault status in motors and pumps.

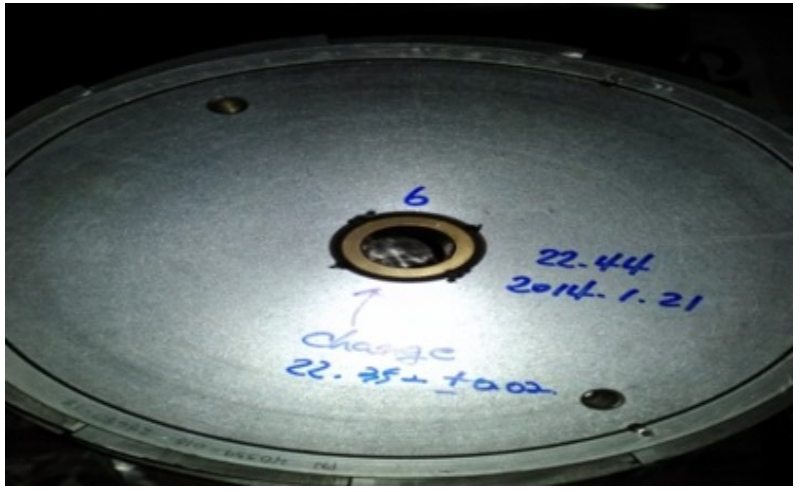

Figure 3. Bushing abrasion status of pump.

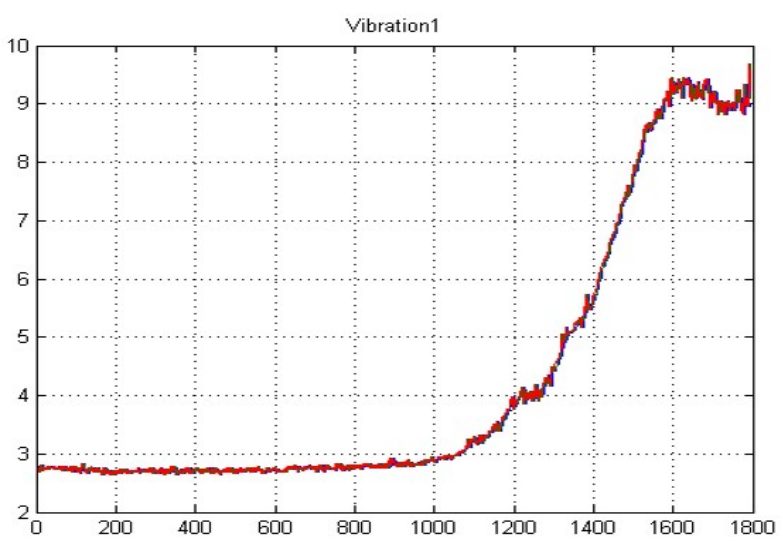

Figure 4. Time series of vibration for the bushing abrasion status.

Hence we use only the data acquisition system to collect the current, RPM, and vibration signal data.

\subsection{Row Data for Analysis of Current and Vibration}

Figure 3 shows the bushing abrasion status. The reference is $22.44 \mathrm{~mm}$ but the abrasion is $22.35 \pm 0.02 \mathrm{~mm}$. From this situation, we obtain the vibration data and current data from pump and motor, respectively.

\subsubsection{Vibration data}

Figure 4 shows the vibration data extracted for the bushing abrasion status from the vibration sensor of the pump as shown in Figure 1. Figure 4 shows the time series that are composed as a single variable of one-dimensional vibration data acquired from pump. 


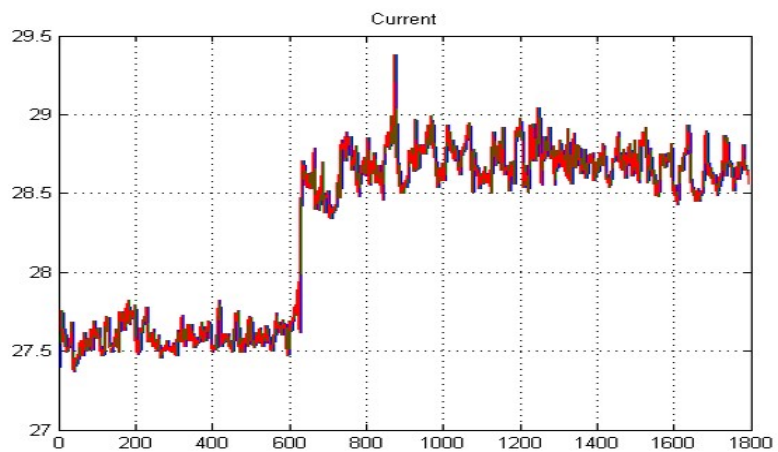

Figure 5. Time series of current for the bushing abrasion status.

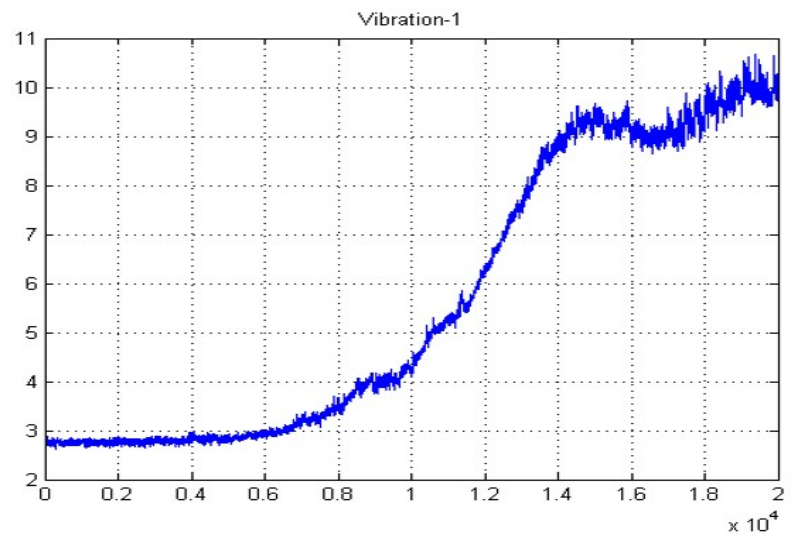

Figure 6. Time series of vibration for the bushing abrasion status after pre-processing.

\subsubsection{Current data}

Figure 5 shows the current data extracted from the motor, which is located under the pump as shown in Figure 2. Figure 5 shows the time series that are composed as a single variable of onedimensional current data acquired from the motor.

\subsection{Data Pre-processing}

The vibration and current data shown in Figure 4 and Figure 5 contain noise. In order to obtain data with excellent qualification, we must eliminate the noise from the data. Using filter and software processing, we achieve the elimination of noise. Hence, we obtain Figure 6 and 7 after we eliminate the noise from time series of vibration and current from Figure 4 and 5, respectively.

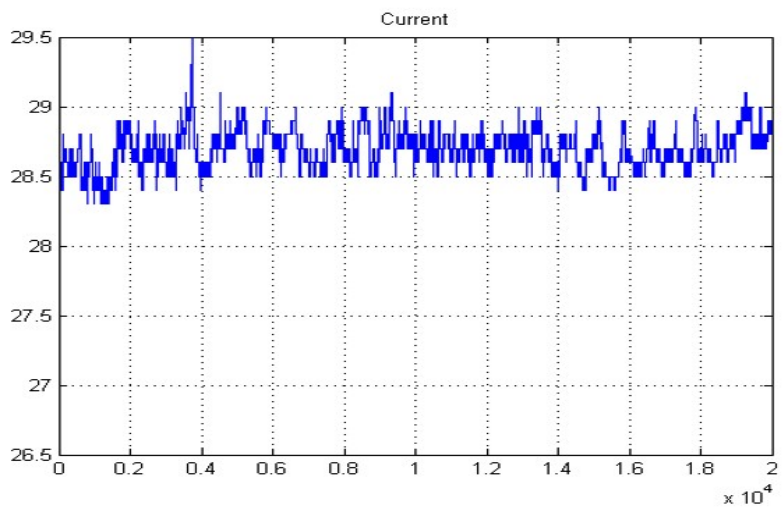

Figure 7. Time series of current for the bushing abrasion status after pre-processing.

\section{State Reconstruction Using Takens' Embed- ding Method}

Because we cannot judge between the normal and fault status using one-dimensional time series of vibration and current data for the pump and motor, we need to transform the time series into a two-dimensional phase portrait. In order to reconstruct a two dimensional phase portrait, we apply an embedding method proposed by Taken [5]. The embedding method refers to the process by which a representation of the attractor is able to reconstruct the state from a set of scalar time series. The form of such reconstructed states is given by

$$
\mathrm{X}_{\mathrm{t}}=[\mathrm{x}(\mathrm{t}), \mathrm{x}(\mathrm{t}+\tau), \cdots \mathrm{x}(\mathrm{t}+(\mathrm{m}-1) \tau)]
$$

where $\mathrm{x}(\mathrm{t})$ is the vibration and current of the bushing abrasion status of the pump and motor. $\tau$ is a delay time, and $\mathrm{m}$ is an embedding dimension. A key factor is choosing the delay time and embedding dimension to reconstruct the phase portrait from the time series. Hence, we choose the value 3 for $\tau$ using an autocorrelation time and sample interval between Poincare sections. We also choose 3 as the embedding dimension. The flow chart of total processing is represented in Figure 8.

Although there is no difference in the time series between prefiltering and post-filtering for the vibration and current signals, we recognize that there is a complete difference in the phase portrait between pre-filtering and post-filtering for both the vibration and current signals of the pump and motor. 


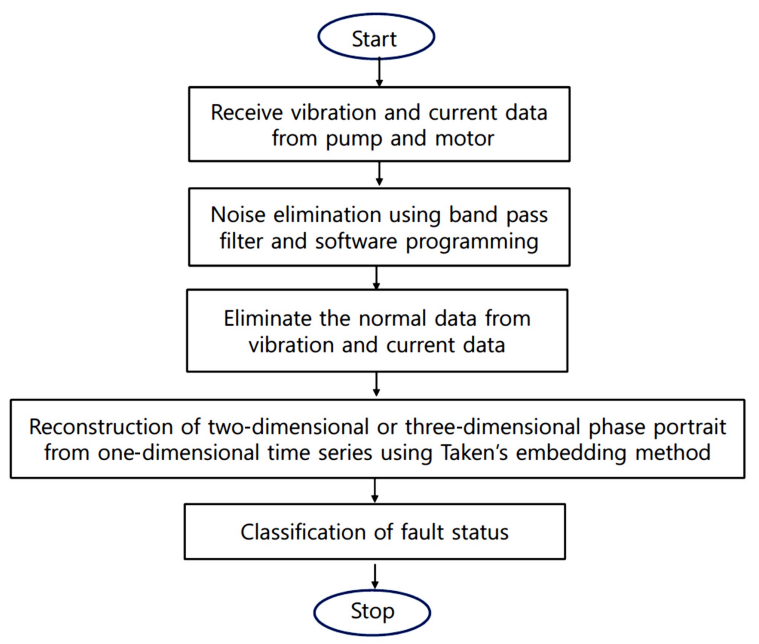

Figure 8. Flow chart for total processing.

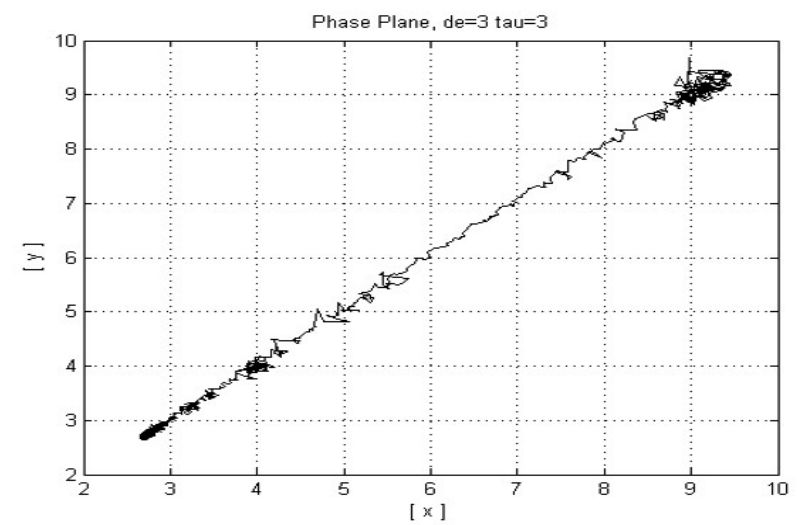

Figure 9. Phase portrait of vibration for the bushing abrasion status before pre-processing.

\section{Conclusion}

In this paper, we measured the current and vibration signals of one-dimensional time series that occur in a motor and pump, respectively. These are representative of rotary machines and pumping machines. We also eliminated unnecessary components such as noise by preprocessing the current and vibration signals. Then, in order to diagnose the fault signal for the pump or motor, we transformed the one-dimensional current and vibration signals to a phase portrait of two or three dimensions using Takens' embedding method. After this transformation, we reviewed the variation of pattern according to the fault signal.

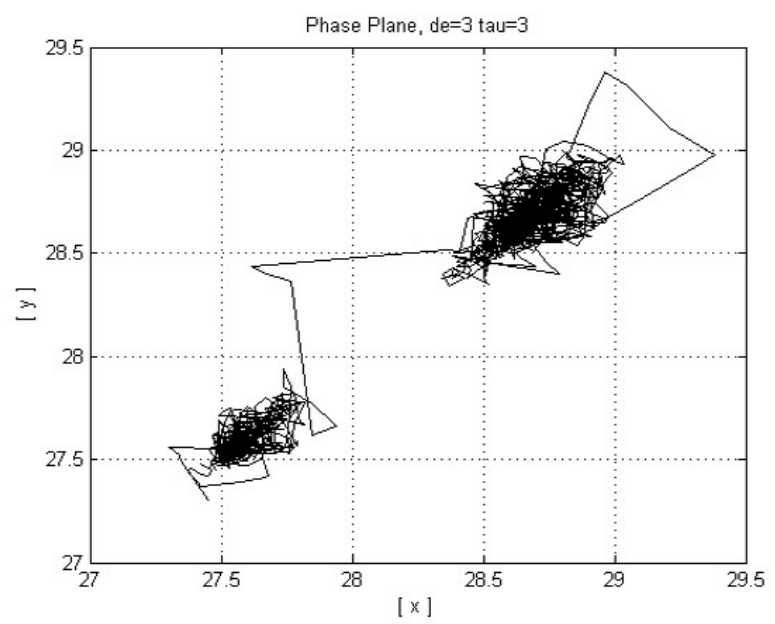

Figure 10. Phase portrait of vibration for the bushing abrasion status after pre-processing.

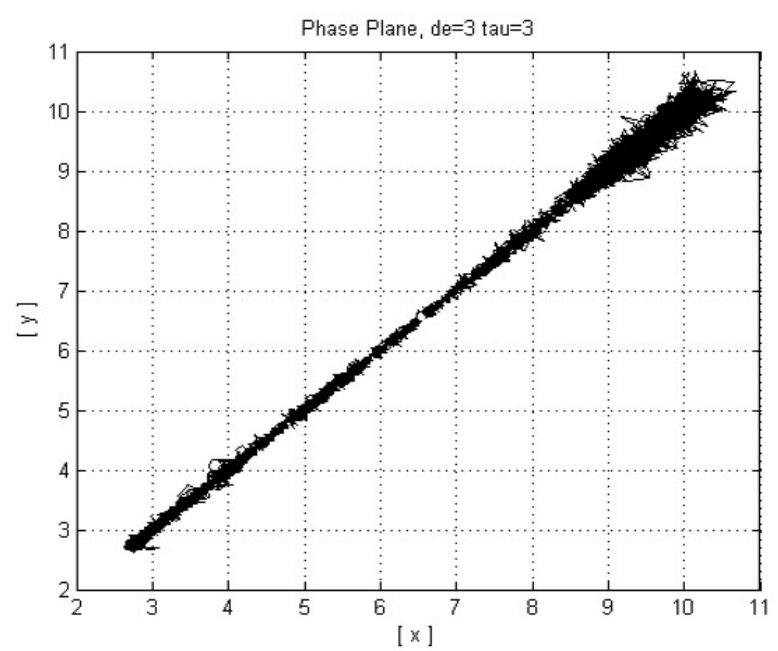

Figure 11. Phase portrait of current for the bushing abrasion status before pre-processing.

\section{Acknowledgment}

This study was supported by the Ministry of Knowledge Economy (MKE) through the Regional Innovation Centre Programme.

This work (Grant No. C0220-390) was supported by the Business for Academic-Industrial Cooperative Establishments funded by the Korea Small and Medium Business Administration in 2014. 


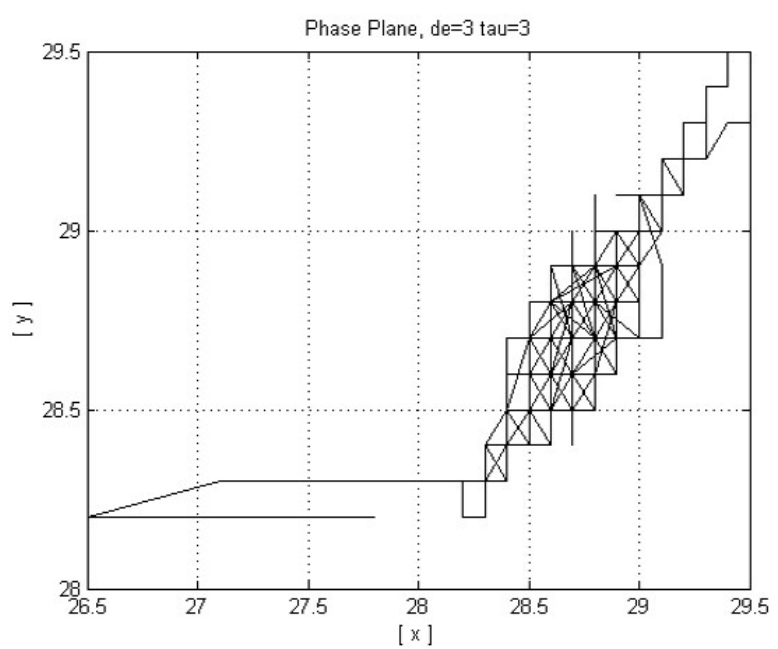

Figure 12. Phase portrait of vibration for the bushing abrasion status after pre-processing.

\section{References}

[1] Colby. R, Mottier. S, Francois, M, and Miller. T.J.E, "Vibration modes and acoustic noise in a four-phase switched reluctance motor,' IEEE TRANSACTIONS ON INDUSTRY APPLICATIONS, vol. 32, no. 6, pp. 1357-1364, 1996.

[2] Nandi. S, Toliyat. H.A, and Xiaodong. L, "Condition monitoring and fault diagnosis of electrical motors-a review," IEEE Transactions on Energy Conversion, vol. 20, no. 4, pp. 719-729, 2005.

[3] Razik. H, and Correa.M, "The tracking of induction motor's faulty lines through particle swarm optimization using chaos," Industrial Technology (ICIT), 2010 IEEE International Conference on Vi a del, pp.1245-1250, Mar, 14-17 March 2010.

[4] Vas. P, Parameter Estimation, Condition Monitoring, and Diagnosis of Electrical Machines, 1993, Clarendon.

[5] Kliman, G.B, and Stein, J,"Induction motor fault detection via passive current monitoring", Proc. Int. Conf. Electrical Machines, pp.13 -17 1990.

[6] Singh, G.K, and Kazzazb, S.A.S.A, "Induction machine drive condition monitoring and diagnostic research survey," in Electric Power Systems Research, vol. 64, no. 2, pp. 145-158, Feb. 2003

[7] Benbouzid, M.E.H, "A review of induction motors signature analysis as a medium for faults detection," in IEEE Trans. Ind. Electron., vol. 47, no. 5, pp. 984993, Oct. 2000.

[8] Thomson, W.T, Deans, N.D, Leonard, R.A, and Milne A.J., "Monitoring Strategy for Discriminating Between Different Types of Rotor Defects in Induction Motors," in Proc. 18th Power engineering Conference, April 1983.

[9] Kliman, G.B, Koegl, R.A, Stein, J, Koegl, R.A, Endicott, R.D and Madden, M.W, "Noninvasive detection of broken rotor bars in operating induction motors," in IEEE Trans. Energy Convers., vol. 3, no. 4, pp. 873 879, Dec. 1988.

[10] Hwang, S and Bae, Y., "Diagnosis of power supply using time-series of infrared camera," J. of the Korea Institute of Electronic Communication Science, 2012, vol. 7, no. 6, pp. 1443-1447.

[11] Bae, Y., "Diagnosis of power supply by analysis of chaotic nonlinear dynamics," J. of the Korea Institute of Electronic Communication Science, 2013, vol. 8, no. 1, pp. 113-1119.

[12] Takens. F, "Detecting strange attractors in turbulence, in dynamical system and turbulence," Lecture notes in Mathematics, 1981, 898, pp. 363-381.

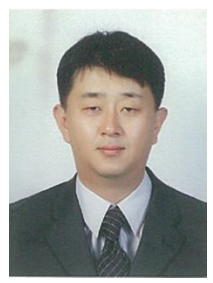

Young-Ok Jung graduate the Dong myoung University, Korea,. He worked at Arkema during 2007-2012, and now working at Air Liquide Korea from 2012 by electrical engineer. He is currently studying at the Division of Electrical, Electronic Communication and Computer Engineering, Chonnam National University, Yeosu, Korea. His research interests include nonlinear dynamics, chaos dynamics, robot control, intelligent system and motor control. Tel: +82-51-264-7000, Fax: +82-51-264-7601

E-mail: Younok.jung@airliquide.com 


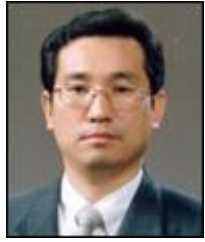

Youngchul Bae received his B.S., M.S., and Ph.D from the Department of Electrical Engineering, Kwangwoon University, Korea, in 1984, 1985, and 1997, respectively. He worked at Korea Electric Power Company (KEPCO) during 1986-1991, and also worked at Korea Institute of Science and Technology Information (KISTI) during 1991-1997 as a senior researcher. He is currently professor at the Division of Electrical, Electronic Communication and Computer Engineering, Chonnam National University, Yeosu, Korea. His research interests include nonlinear dynamics, chaos dynamics, robot control, intelligent system and motor control. $\mathrm{He}$ is member of KIEE, KIECS and KIIS.

Tel: +82-61-659-7315, Fax: +82-61-659-7310

E-mail:ycbae@chonnam.ac.kr 